\title{
Debris flow modelling accounting for large boulder transport
}

\author{
C. Martinez ${ }^{1}$, F. Miralles-Wilhelm ${ }^{1}$ \& R. Garcia-Martinez ${ }^{2}$ \\ ${ }^{I}$ Department of Civil and Environmental Engineering, \\ Florida International University, USA \\ ${ }^{2}$ Applied Research Center, Florida International University and \\ FLO-2D Software, Inc., USA
}

\begin{abstract}
We present a quasi three-dimensional numerical model to simulate stony debris flows, considering a continuum fluid phase of water and fine sediments, and a non-continuum phase of large particles, such as boulders. Large particles are treated in a Lagrangian frame of reference using the Discrete Element Method in three dimensions. The fluid phase is governed by the depth-averaged Navier-Stokes equations in two horizontal dimensions and is solved by the Finite Element Method. The model simulates particle-particle collisions and wall-particle collisions, taking into account that particles are immersed in the fluid. Bingham and Cross rheological models are used for the continuum phase. Both formulations provide stable results, even in the range of very low shear rates. The Bingham formulation is better able to simulate the stopping stage of the fluid. The results of the numerical simulations are compared with data from laboratory experiments on a flume-fan model. The results show that the model is capable of simulating the motion of big particles moving in the fluid flow, handling dense particulate flows that avoid overlapping among particles. An application to simulate a debris flow event that occurred in Northern Venezuela in 1999 shows that the model replicates well the main observed boulder accumulation areas.
\end{abstract}

Keywords: debris flow, mud flow, boulders transport, Eulerian and Lagrangian formulation, finite element method, discrete element method. 


\section{Introduction}

Debris flow is a frequent phenomenon in mountainous regions. It occurs when masses of poorly sorted sediments, rocks and fine material, agitated and mixed with water, surge down slopes in response to water flow and gravitational attraction. A typical surge of debris flow has a steep front or "head" with the densest slurry, the highest concentration of boulders and the greatest depth. A progressively more dilute and shallower tail follows this head.

Reviews presented by Iverson [1], exhaustively describe the physical aspects of debris flow motion and clearly divide previous debris flow research into two distinct categories. The first, based upon the pioneering work of Johnson [2], assumes that debris flow behaves as a viscoplastic continuum. This model describes a single-phase material that remains rigid unless stresses exceed a threshold value: the plastic yield stress. Various rheological models have been proposed, derived from experimental results or from theoretical considerations, such as the Bingham model [3], the Cross model [4], and the quadratic model proposed by O'Brien and Julien [5]. The Bingham plastic model is the most commonly used in practice.

The second approach has focus on the mechanics of granular materials. Based upon the findings of Bagnold [6], two-phase models have been developed by several authors, such as Takahashi [7] and Iverson [1]. These models explicitly account for solid and fluid volume fractions and mass changes respectively.

Despite of the considerable progress over the past few years, the flow dynamics and internal processes of debris flows are still challenging in many aspects. In particular, there are many factors related to the movement and interaction of individual boulders and coarse sediments that have not been fully addressed in previous works. Asmar et al. [8] introduced the Discrete Element Method (DEM) to simulate the motion of solid particles in debris flows. DEM is a numerical method to model dry granular flows where each particle is traced individually in a Lagrangian frame of reference by solving Newton's equation of motion.

This paper describes the development of a quasi three-dimensional model to simulate stony debris flows, considering a continuum fluid phase, and large sediment particles, such as boulders, as a non-continuum phase. Large particles are treated in a Lagrangian frame of reference using DEM, and the fluid phase composed by water and fine sediments is modelled with an Eulerian approach using the depth-averaged Navier-Stokes equations in two dimensions. Bingham and Cross rheological models are used for the continuum phase. Particle's equations of motion are fully three-dimensional. The model is tested with laboratory experiments and with a real application.

\section{Governing equations}

The flow domain is divided in computational cells with triangular base and depth $H$, as shown in Figure 1. 


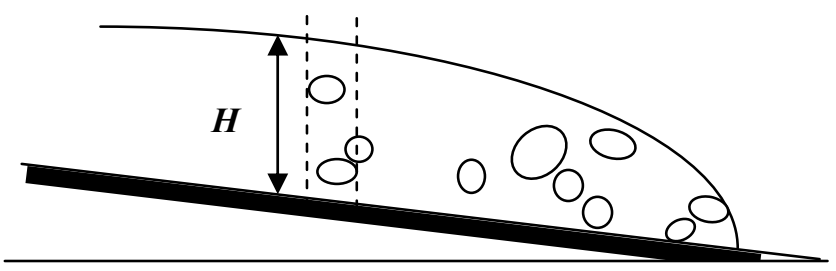

Figure 1: $\quad$ Schematic representation of debris flow with large solid particles.

Assuming non-Newtonian and incompressible fluid phase, the depth averaged continuity and momentum equations in Cartesian coordinates can be written as follows:

$$
\begin{gathered}
\frac{\partial H}{\partial t}+\frac{\partial(\bar{u} H)}{\partial x}+\frac{\partial(\bar{v} H)}{\partial y}=0 \\
\frac{1}{g} \frac{\partial \bar{u}}{\partial t}+\frac{\bar{u}}{g} \frac{\partial \bar{u}}{\partial x}+\frac{\bar{v}}{g} \frac{\partial \bar{u}}{\partial y}+\frac{\partial \eta}{\partial x}+\frac{F_{D x}}{\rho g}+S_{f x}=0 \\
\frac{1}{g} \frac{\partial \bar{v}}{\partial t}+\frac{\bar{u}}{g} \frac{\partial \bar{v}}{\partial x}+\frac{\bar{v}}{g} \frac{\partial \bar{v}}{\partial y}+\frac{\partial \eta}{\partial y}+\frac{F_{D y}}{\rho g}+S_{f y}=0
\end{gathered}
$$

where $H$ is the water depth, $\eta$ is the free-surface elevation, $\bar{u}$ and $\bar{v}$ are the depth averaged velocities in $x$ and $y$ directions respectively, $g$ is the gravitational acceleration and $\rho$ is fluid density. $\mathbf{F}_{D}$ represents the fluid-solid interaction force exerted on the fluid by particles through the fluid drag force.), this force is evaluated as:

$$
\mathbf{F}_{D}=\frac{\sum_{i=1}^{n} \mathbf{F}_{F D i}}{\Delta V}
$$

where $\mathbf{F}_{F D}$ is the fluid drag force on each particle $i, \Delta V$ is the volume of the computational cell and $n$ is the number of particles in the cell. $S_{f x}$ and $S_{f y}$ are the depth integrated stress terms that depend on the rheological formulation used to model the slurry.

Assuming a Bingham rheological model and Manning's formula, as proposed by O'Brien and Julien [5], the stress terms for the fluid can be expressed as 


$$
\begin{gathered}
S_{f x}=\frac{\tau y}{\rho g H}+\frac{3 \mu \bar{u}}{\rho g H^{2}}+\frac{N^{2} \bar{u}^{2}}{H^{4 / 3}} \\
S_{f y}=\frac{\tau y}{\rho g H}+\frac{3 \mu \bar{v}}{\rho g H^{2}}+\frac{N^{2} \bar{v}^{2}}{H^{4 / 3}}
\end{gathered}
$$

where $N$ is the Manning roughness coefficient.

The fluid dynamic viscosity $\mu$ and yield stress $\tau_{y}$, are determined as functions of the volume sediment concentration $C v$, using the relationships proposed by O'Brien and Julien [9]:

$$
\begin{gathered}
\mu=\alpha_{1} e^{\beta_{1} \bar{c}} \\
\tau_{y}=\alpha_{2} e^{\beta_{2} \bar{c}}
\end{gathered}
$$

in which $\alpha_{1}, \beta_{1}, \alpha_{2}$ and $\beta_{2}$ are empirical coefficients obtained by data correlation in a number of experiments with various sediment mixtures. Using a quadratic formulation combined with the Cross rheological model, the stress terms for the fluid are expressed as

$$
\begin{gathered}
S_{f x}=\frac{\mu_{e f f} \dot{\gamma}}{\rho g H}+\frac{N^{2} \bar{u}^{2}}{H^{4 / 3}} \text { with } \dot{\gamma}=\frac{3 \bar{u}}{H} \\
S_{f y}=\frac{\mu_{e f f} \dot{\gamma}}{\rho g H}+\frac{N^{2} \bar{v}^{2}}{H^{4 / 3}} \text { with } \dot{\gamma}=\frac{3 \bar{v}}{H}
\end{gathered}
$$

where $\mu_{\text {eff }}$ is the effective viscosity of the fluid defined by:

$$
\mu_{\text {eff }}=\frac{\mu_{0}+\mu_{\infty} K_{B} \dot{\gamma}}{1+K_{B} \dot{\gamma}}
$$

with $K_{B}=\frac{\mu_{0}}{\tau_{y}}, \mu_{\infty}=\mu$ and $\mu_{0}=10^{3} \mu$

In the solid phase, spherical particles of different diameters are considered. Particle trajectories are tracked using Newton's second law and the considering gravity, buoyancy, fluid drag and collision forces. 


$$
m_{i} \frac{d \mathbf{v}}{d t}=\sum \mathbf{F}_{E}+\sum \mathbf{F}_{N}+\sum \mathbf{F}_{T}
$$

The external force $\mathbf{F}_{E}$ is given by

$$
\mathbf{F}_{E}=\mathbf{F}_{B}+\mathbf{F}_{F D}
$$

The expression to compute the net force acting on the particle due to gravitational effects is

$$
\mathbf{F}_{B}=\frac{4}{3} \pi R^{3}\left(\rho-\rho_{p}\right) \mathbf{g}
$$

where $R$ is the particle radius and $\rho_{p}$ is the particle density.

The expression for the drag on particles in viscous fluid is given by

$$
\mathbf{F}_{F D}=\frac{1}{2} \pi R^{2} C_{d} \rho|\mathbf{u}-\mathbf{v}|(\mathbf{u}-\mathbf{v})
$$

where $C_{d}$ is the drag coefficient, $\mathbf{u}$ is the fluid velocity vector at the particle location, and $\mathbf{v}$ is the particle velocity vector.

The last two terms in equation (12) represent the collision forces or contact forces among particles. Based on the simplified model that uses a springdashpot-slider system to represent particle interactions [8], the normal contact force and the tangential contact force are evaluated as

$$
\begin{gathered}
\mathbf{F}_{N}=\mathbf{F}_{N C}+\mathbf{F}_{N D} \\
\mathbf{F}_{T}=\mathbf{F}_{T C}+\mathbf{F}_{T D}
\end{gathered}
$$

The normal contact force $\mathrm{F}_{N C}$ is calculated using a Hook's linear spring relationship,

$$
\mathbf{F}_{N C}=K_{N} \delta_{N}
$$

where $K_{N}$ is the normal contact stiffness and $\delta_{N}$ is the displacement (overlap) between particles $i$ and $j$.

The normal damping force $\mathrm{F}_{N D}$ is also calculated using a linear relation given by

$$
\mathbf{F}_{N D}=C_{N} v_{N}
$$


where $v_{N}$ is the normal component of the relative velocity between particles and $C_{N}$ is the normal damping coefficient. This constant $C_{N}$ is chosen to give a required coefficient of restitution $\beta$, defined as the ratio of the normal component of the relative velocities before and after collision.

The tangential contact force, FTC, represents the friction force and it is constrained by the Coulomb frictional limit, at which point the particles begin to slide over each other. Prior to sliding, the tangential contact force is calculated using a linear spring relationship,

$$
\mathbf{F}_{T C}=K_{T} \delta_{T}
$$

where $K_{T}$ is the tangential stiffness coefficient, and $\delta_{T}$ is the total tangential displacement between the surfaces of particles $i$ and $j$ since their initial contact. When $K_{T} \delta_{T}$ exceeds the frictional limit force $\mu_{f} F_{N C}$, particle sliding occurs. The sliding condition is defined as

$$
\mathbf{F}_{T C}=\mu_{f} \mathbf{F}_{N C}
$$

where $\mu_{f}$ is the dynamic friction coefficient.

The tangential damping force $\mathbf{F}_{T D}$ is not included in this model, since it is assumed that once sliding occurs, damping is accounted for from friction.

Also, particle rotation is not considered. Fluid governing equations (1-3) are solved by the Galerkin Finite Element method using three-node triangular elements. To solve the resulting system of ordinary differential equation, the model applies a four-step time stepping scheme and a selective lumping method, as described by Garcia-Martinez et al. [10].

Forces on each particle are evaluated at each time step, and the acceleration of the particle is computed from the particle governing equation, which is then integrated to find velocity and displacement of each particle.

\section{Results}

A series of experiments were carried out in a laboratory flume, using homogeneous fluid and fine sediment mixtures for the continuum phase and spherical marbles for the discrete phase. The experiments were performed in a $1.9 \mathrm{~m}$ long, $0.19 \mathrm{~m}$ wide, Plexiglas walled flume, with adjustable slope. The downstream part of the flume was connected to a wood horizontal platform, 0.75 $\mathrm{m}$ long and $0.95 \mathrm{~m}$ wide. A dam-break type of flow was initiated by an abrupt removal of a gate releasing mixtures from a $0.40 \mathrm{~m}$ long reservoir situated on the upstream part of the flume. Water-clay mixtures were used in all the experiments, with volume sediment concentration $23.5 \%$ and $26.5 \%$. For preparation of the mixtures, kaolinite clay with specific unit weight of 2.77 was used. Fluid density was measured in the laboratory and rheological parameters $\mu$ and $\tau_{y}$ were determined using equations (7) and (8) in which parameters are $\alpha_{1}=$ $0.621 \times 10^{-3}, \beta_{1}=17.3, \alpha_{2}=0.002$ and $\beta_{2}=40.2$. 
Table 1: $\quad$ Rheological properties of experimental fluids.

\begin{tabular}{|c|c|c|c|}
\hline$C_{v}(\%)$ & $\rho\left(K g / \mathbf{m}^{3}\right)$ & $\mu(P a . s)$ & $\tau_{y}(P a)$ \\
\hline 23.5 & 1410 & 0.0362 & 25.34 \\
\hline 26.5 & 1460 & 0.0608 & 84.64 \\
\hline
\end{tabular}

\subsection{Experiment 1}

In this experiment, the flow of a fluid of $23.5 \%$ volume concentration was studied. The flume bottom slope was set to $9.54^{\circ}$ and the initial volume released was $6.4 \mathrm{~L}$. The objective of this test was to study the spreading of the fluid in the fan and the particle interaction with the fluid.

14 particles, with diameter $D=2.5 \mathrm{~cm}$ and density $\rho_{p}=2500 \mathrm{Kg} / \mathrm{m}^{3}$, were placed over a small piece of wood inside the mud reservoir, just behind the gate. By the time the fluid was released, the piece of wood was quickly removed, so that the particles could start their movement along the flume with the fluid. At early times after the release, particles travelled downstream on a parabolic formation across the flume following the parabolic velocity profile. However, as the flow moved downstream, particles tended to move to the flume sides.

It is important to mention the effect of the boundary condition for velocity on the sidewalls. Typically, this condition should be a no-slip condition expressed as $\vec{u}=0$ at the wall. However, in reality, the velocity gradient near the wall is large and the velocity near a wall quickly becomes non-zero. Therefore, in practical applications, this condition becomes very restrictive, causing unrealistic delay of the flow. For this simulation, a more relaxed boundary condition was tested where, the normal velocity is $u_{N}=0$, and the tangential velocity is $u_{T}=0.9(\vec{u} \cdot \vec{t})$.

Figure 2 compares final position of particles obtained numerically, with observed final position for particles $(\mathrm{t}=10 \mathrm{~s})$. The flood extent and the final particle locations obtained numerically replicates reasonably well the experiment. In the numerical results there is some delay on the particles positioned close to the walls that is attributed to the calibration of the boundary condition for tangential velocity at the walls. If a full slip condition is imposed, then the transversal velocity profile disappears across the channel, generating unrealistic results as shown in Figure 3.

\subsection{Experiment 2}

In this experiment, a mixture of volumetric concentration of $26.5 \%$ was studied. In this case, the flume bottom slope was increased to $10.7^{\circ}$ and the initial volume released was $11.1 \mathrm{~L}$. The objective of this test was to study the spreading of the fluid and study particle movement into a mixture with higher clay concentration.

In this experiment, the velocity of the front wave is basically constant until reaching $1.6 \mathrm{~m}$, from this point the celerity of the wave decreases abruptly, taking about $40 \mathrm{~s}$ for the fluid to stop completely. 

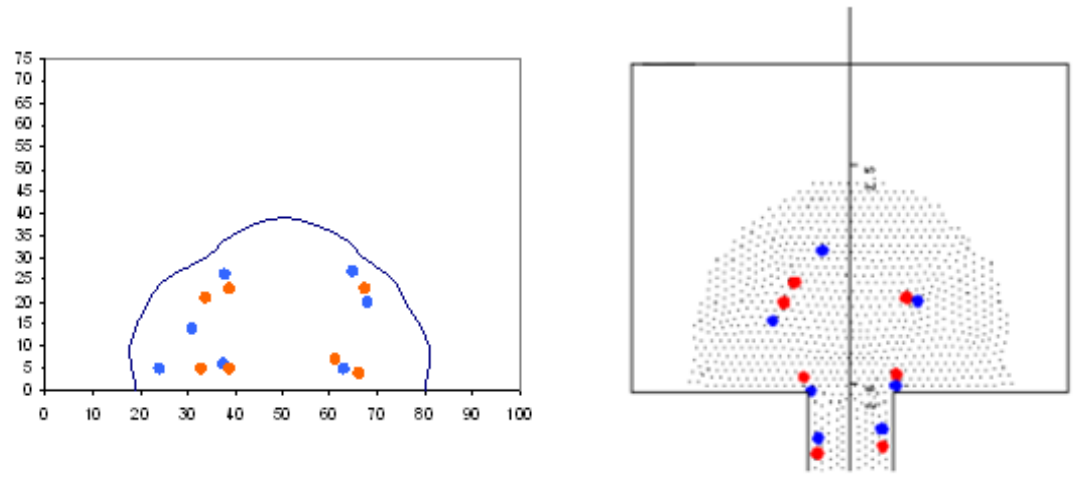

Figure 2: Exp. 1, final position of particles, (a) experimental data (b) numerical solution.

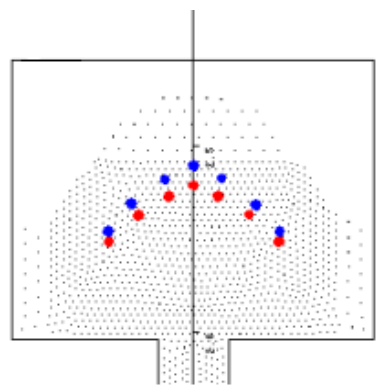

Figure 3: Exp. 1, final position of particles with fully slip boundary condition.

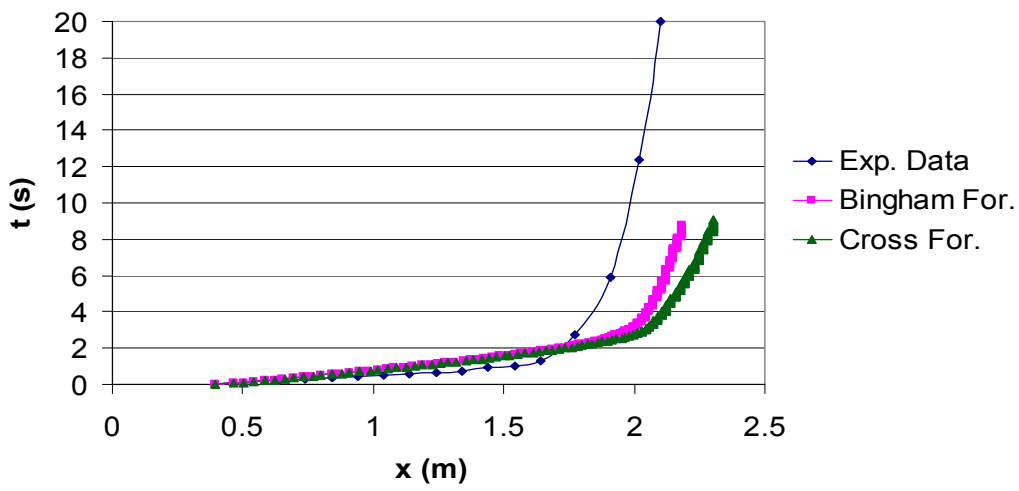

Figure 4: $\quad$ Exp. 2, spreading relation. 
Figure 4 shows the spreading relation in the longitudinal direction for this experiment. This relation is compared with numerical results obtained using Bingham rheological model and using Cross rheological model.

Although both rheological formulations produce very similar results, they are not totally capable of resembling the spreading of the flow. However, they show a final fluid extent very similar to the experimental one.

In this experiment 14 particles were placed on the fluid in a similar manner that was done in the previous experiment. In this case, particles depicted the velocity profile shape at early times of the experiment; and as the flow progress down-stream, particles keep the parabolic distribution.

Figure 5 compares the final particle positions obtained numerically against final observed particle location. Note that the model is able to replicate that some particles lag behind close to the flume wall and that the general location of the particles on the alluvial fan is very close to the observed locations.
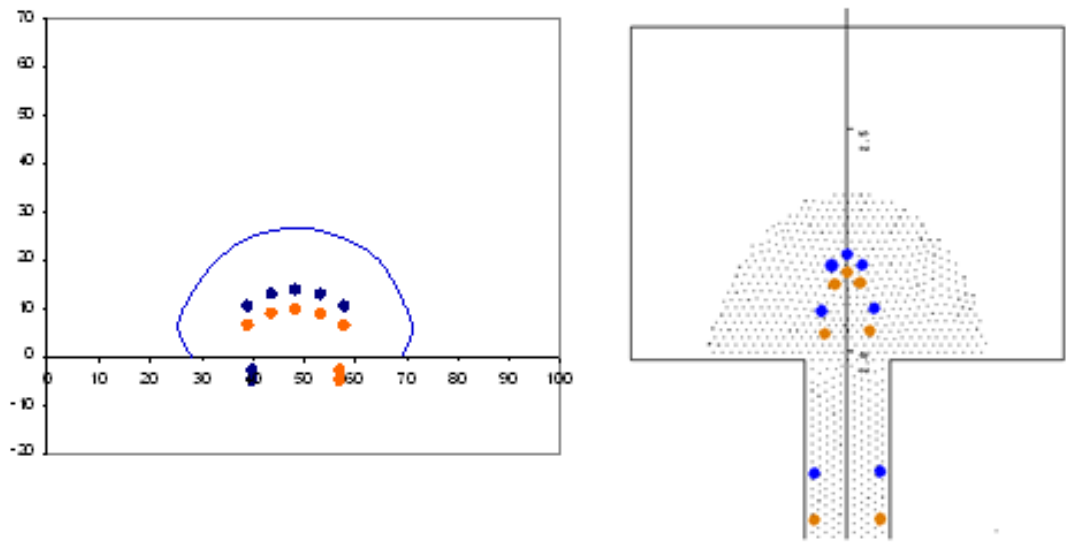

Figure 5: Exp. 2, final position of particles, (a) experimental data (b) numerical sol.

\subsection{Model preliminary application: Venezuela's 1999 alluvial fan debris flooding event}

Heavy rainfall from a storm on December 14-16, 1999, triggered thousands of shallow landslides on steep slopes of Cerro El Avila, north of Caracas, Venezuela, and caused flooding and massive debris flows in the channels of major drainages that severely damaged coastal communities along the Caribbean Sea. The largest fan on this area is that of San Julián River at Caraballeda, shown in Figure 6. This fan was one of the most heavily damaged areas in the event. The thickness of sediment deposition, maximum size of transported boulders, and size of inundated area were all notably larger in this drainage in comparison to the other close watersheds. 


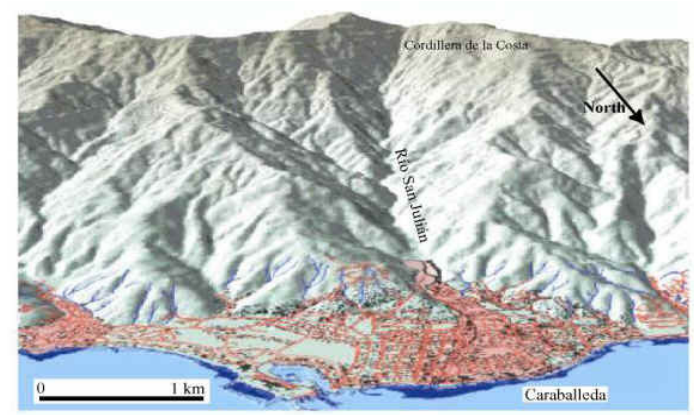

Figure 6: Caraballeda Alluvial Fan, Vargas, Venezuela.

The US Geological Survey studied the affected area [11], measuring slope, deposit thickness, and boulder size from the fan apex to the distal end of the fan near the coastline. Data was used to map the distribution and thickness of deposits and to draw contours of maximum boulder size, as shown in Figure 7.

The numerical simulation was performed using a finite element mesh with 22,500 triangular elements. The element characteristic size was $12 \mathrm{~m}$ on average. At the fan apex, a 500 year-return period hydrograph was used as flow input with an average volume sediment concentration of $C_{v}=0.3$. Fluid properties are $\rho=$ $1531 \mathrm{Kg} / \mathrm{m}^{3}, \mu=0.11 \mathrm{~Pa} . \mathrm{s}, \tau_{y}=105 \mathrm{~Pa}$. During the simulation, 1600 boulders with sizes ranging from $1 \mathrm{~m}$ to $6 \mathrm{~m}$ diameter were included in the event. Density for the boulders is $\rho=2600 \mathrm{Kg} / \mathrm{m}^{3}$, equal to the density of Gneiss boulders, the type of boulders mostly found in the area by the USGS.

Figure 7 shows boulder positions after 6 hours of simulation in comparison with contours of maximum boulder size given by USGS. According to USGS, for station $\mathrm{S} 1$ the mean nominal diameter was $1 \mathrm{~m}$, while some larger boulders are deposited slightly further down the fan towards station S2, with $3.5 \mathrm{~m}$ nominal diameter. For comparison, boulders deposited at station S3 and S4 had mean nominal diameter of $3 \mathrm{~m}$, and boulders deposited at station S5 had average nominal diameter of $5 \mathrm{~m}$. Figure 7 (b) shows the final distribution of boulders obtained numerically, where it can be seen that the model predicts reasonable boulder locations as compared with the field data.

\section{Conclusions}

This work describes the development and application of a quasi threedimensional two-phase model to simulate debris flows, considering large sediment particles, such as boulders. The continuum non-Newtonian phase is solved by the finite element method in 2D and the particle transport with the Discrete Element Method in 3D. The model is able to replicate fluid and particle transport when compared against several experiments in a laboratory flume-fan, 


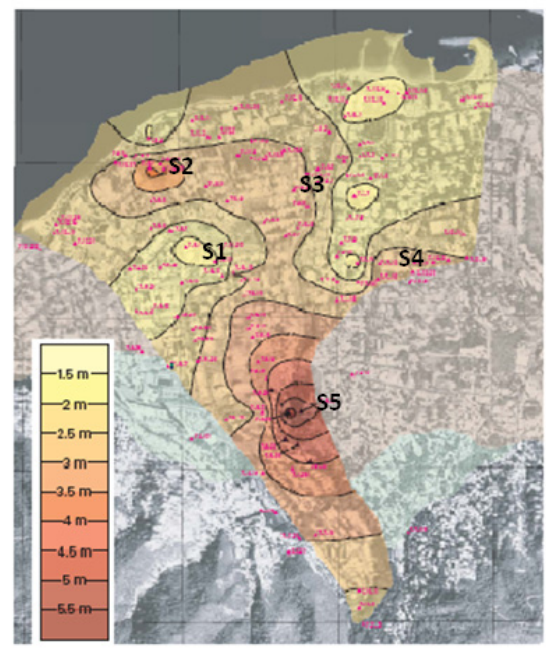

(a)

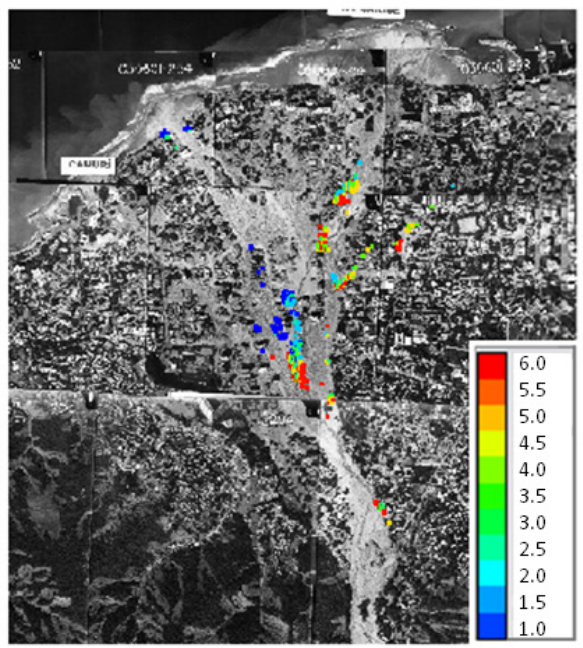

(b)

Figure 7: (a) Contours of maximum boulder size at the fan generated from field data. (b) Particle positions at $\mathrm{t}=6.0 \mathrm{~h}$.

including the effect of particle-particle and wall-particle collisions. An application to the well documented debris flow event that occurred in Northern Venezuela in 1999 illustrates the capability of the model to reproduce large scale real events. Results show that the model reasonably approximates the flood extent affected by the debris flow and the observed boulder accumulation areas, including distribution boulders sizes. Future work includes comparison with field events using larger number of boulders to improve predictions.

\section{References}

[1] Iverson, R. M. The physics of debris flows. Rev. of Geophysics, 35, pp. 245-296, 1997b.

[2] Johnson, A. M. A model for debris flow. Ph.D. dissertation. Pennsylvania State University, University Park. 1965.

[3] Bingham, E. C., and Green, H. Paint, a plastic material and not a viscous liquid; the measurement of its mobility and yield value. Proceedings of American Society of Testing Materials, 19, pp. 640-664, 1919.

[4] Barnes, H.A., Hutton J.F., Walters, K. An introduction to rheology. Amsterdam. Elsevier. 1989

[5] O'Brien, J.S. and Julien, P.Y. Physical properties and mechanics of hyperconcentrated sediment flows. ASCE Specialty Conference on the Delineation of Landslides, Debris Flows Hazards, pp. 260-279, 1985.

[6] Bagnold, R. A. Experiments on a gravity-free dispersion of large solid spheres in a Newtonian fluid under shear. Proceedings of the Royal Society of London, 225, pp. 49-63, 1954. 
[7] Takahashi, T. Debris Flows. Rotterdam, Balkema. 1991.

[8] Asmar B. N., Langston, P. A. and Ergenzinger, P. The potential of the Discrete Element Method to simulate debris flow. Debris-flow hazards mitigation: mechanics, prediction and assessment, 1, pp. 435-445, 2003.

[9] O'Brien, J.S. and Julien, P.Y. Laboratory analysis of mudflows properties. J. of Hyd. Eng., 114(8), pp. 877-887, 1988.

[10] García-Martínez, R., Espinoza, R., Valera, E. \& González, M. An explicit two-dimensional finite element model to simulate short and long term bed evolution in alluvial rivers. J. of Hyd. Res., 44 (6), pp. 755-766, 2006.

[11] Wieczorek, G.F., Larsen, M.C., Eaton, L.S., Morgan, B.A. and Blair, J. L. 2001. Debris-flow and flooding hazards associated with the December 1999 storm in coastal Venezuela and strategies for mitigation. U.S. Geological Survey, Open File Report 01-0144. 2001. 